\title{
Is AST/ALT Ratio a Predictor of In-hospital Mortality in Pulmonary Embolism Patients?
}

\author{
Muhammed Necati Murat Aksoy ${ }^{1}$, Fahrettin Turna ${ }^{1}$, Irfan Sahin ${ }^{2}$ and Suret Agac ${ }^{3}$ \\ ${ }^{1}$ Department of Cardiology, Faculty of Medicine, Sakarya University, Sakarya, Turkey \\ ${ }^{2}$ Department of Cardiology, Istanbul Bagcilar Research and Education Hospital, Istanbul, Turkey \\ ${ }^{3}$ Department of Biochemistry, Sakarya University Research and Education Hospital, Sakarya, Turkey
}

\begin{abstract}
Objective: To document the association between serum transaminases and in-hospital mortality in pulmonary embolism (PE) patients.

Study Design: Analytical study.

Place and Duration of Study: Patients treated with acute PE in hospital between January 2011 and December 2019 from Sakarya University Medical School Research and Teaching Hospital, Sakarya, Turkey.

Methodology: Patients with PE were included. Receiver operating characteristics (ROC) analysis was conducted to define a cut-off value for AST/ALT ratio to predict in-hospital death. Simplified pulmonary embolism severity index (sPESI) scores were calculated and the AST/ALT ratio were entered into binary logistic regression analysis with components of sPESI score to document the prognostic significance of as an independent predictor of in-hospital mortality.

Results: 164 acute PE patients were included; of those, 33 (20\%) died in hospital. Deceased patients had higher AST/ALT ratio (median, $1.4 ; 25^{\text {th }}-75^{\text {th }}$ percentile, $1.1-1.8$ ) comparing to patients with discharged home (median, $1.1 ; 25^{\text {th }}-75^{\text {th }}$ percentile, $0.84-1.4$ ) $(p=0.006)$. ROC analysis showed that AST/ALT ratio was an indicator of in-hospital mortality, and the calculated area under the curve was $0.655(95 \% \mathrm{Cl}, 0.547-0.764)$. The cut-off value of 1.3 was associated with a prognostic sensitivity of $61 \%$ and specificity of $65 \%$. Binary logistic regression analysis failed to show AST/ALT ratio as an independent predictor of in-hospital mortality.

Conclusion: AST/ALT ratio predicts in-hospital mortality with acceptable sensitivity and specificity in patients with acute PE and might be used as a biomarker for risk stratification.
\end{abstract}

Key Words: Pulmonary embolism, In-hospital mortality, Transaminases.

How to cite this article: Aksoy MNM, Turna F, Sahin I, Agac S. Is AST/ALT Ratio a Predictor of In-hospital Mortality in Pulmonary Embolism Patients?. J Coll Physicians Surg Pak 2022; 32(02):171-176.

\section{INTRODUCTION}

Pulmonary embolism (PE) is the third most frequent acute cardiovascular disease (CVD) along with myocardial infarction and stroke. ${ }^{1}$ Determining the severity of the clinical situation is of paramount importance and guides physicians in administering life-saving treatments. The primary pathophysiological basis of the clinical picture in severe $\mathrm{PE}$ is right ventricular (RV) pressure overload, which leads to acute RV failure and subsequent circulatory collapse and cardiogenic shock. Therefore, low systolic blood pressure at the time of diagnosis is the key predictor of severe outcomes, including early mortality in the course of the disease. ${ }^{2}$ Furthermore, rising levels of serum myocardial injury biomarkers seem to be related to worse early outcomes. ${ }^{3,4}$

Correspondence to: Dr. Muhammed Necati Murat Aksoy, Department of Cardiology, Sakarya University Medical School Research and Teaching Hospital, Sakarya, Turkey E-mail:draxoy@gmail.com

Received: June 02, 2021; Revised: August 20, 2021;

Accepted: December 06, 2021

DOI: https://doi.org/10.29271/jcpsp.2022.02.171
In theabsence of arterial hypotension, serum biomarkerassessment of RV overload and dysfunction, including B-type natriuretic peptide (BNP) and N-terminal (NT)-proBNP, may also help in risk stratification for early adverse events. ${ }^{5}$ Accordingly, estimation of early mortality risk is generally based on the echocardiographic assessment of RV size and function, laboratory biomarkers such as troponin and natriuretic peptide levels, ${ }^{3,6-8}$ and the patient's hemodynamic profile at the time of presentation. On the other hand, pulmonary embolism severity index (PESI) and simplified PESI (sPESI) scoring systems are currently being used to identify high risk patients in terms of early clinical adverse events and mortality. ${ }^{9,10}$ Both of these scoring systems are helpful to identify low risk PE patients with an acceptable negative predictive value.

According to the aforementioned clinical and laboratory findings, treatment of PE relies on prognostic clinical classification schemes and early mortality risk proposed by various guidelines. Classifying a patient in the high-risk category for early adverse events leads to aggressive treatment strategies such as thrombolytic infusions to restore pulmonary arterial blood flow. In this regard, it was hypothesised that serum biomarkers of liver function, aspartate aminotransferase (AST), and alanine 
aminotransferase (ALT) in particular might help clinicians in further risk stratification as pulmonary embolism and subsequent acute right heart failure leads to alterations in liver function tests. ${ }^{11}$

This aim of this study was to investigate whether the pattern of liver enzyme elevations presented as AST/ALT ratio is associated with early mortality risk or not in PE patients.

\section{METHODOLOGY}

This study was approved by the Ethics Committee of Sakarya University School of Medicine, Istanbul, Turkey and was conducted in Sakarya University Medical School Research and Teaching Hospital. Databases from both hospitals, including patients treated with the primary diagnosis of PE between 2011-2019, were reviewed. Detection of thrombus in pulmonary arterial bed with contrast-enhanced computed tomography was mandatory to be included in the study. Of those 635 patients treated with PE diagnosis, 164 patients were included in this retrospective analysis. Patients who were asymptomatic and diagnosed with PE during testing for another primary diagnose, patients without AST or ALT levels recordings on admission, and patients with known acute or chronic hepatic diseases were excluded. Patients with a current or recent history of drug usage, which alters serum transaminase levels and affects liver functions, were also excluded.

Echocardiographic parameters, which were obtained in the first 24 hours after the presentation, were noted. Hemodynamic parameters (blood pressure, pulse rate) were recorded from patients' first contact reports from the Emergency Department or elsewhere. Laboratory data, including AST/ALT, creatine, Ddimer, and troponin levels, were obtained from their first recordings of the admitted ward. sPESI scores were calculated from patients' history and hemodynamic recordings. Patients' demographic data and medical history were acquired from patients themselves or their first-degree relatives if the patient's clinical condition did not allow for appropriate verbal contact.

All patients received either intravenous heparin infusion or subcutaneous low molecular weight heparin as first line therapy adjusted according to their activated partial thromboplastin time or weight. The attending physician decided on intravenous thrombolytic administration according to the latest clinical guidelines and patients' clinical situation. The primary endpoint was death from any cause during follow-up in the hospital. This protocol was approved by local Ethics Committees of participating sites.

One sample Kolmogorov-Smirnov test was used to assess the normality of distribution for continuous variables. Data are expressed as mean \pm standard deviation (SD) for normally distributed continuous variables, as median and interquartile ranges for skew-distributed continuous variables, and as frequencies for categorical variables. Patients were dichotomised according to the major clinical endpoint of death. The means for normally distributed continuous variables were compared by independent-samples t-test. Skew-distributed continuous variables were compared, using a Mann-Whitney Utest. The prognostic significance of the AST/ALT ratio was assessed with binary logistic regression analysis. Correlation betweenAST/ALTratio andsPESI score was analysed with Spearman's correlation analysis. Troponin T level and AST/ALT ratio were entered to logistic regression model along with classical prognostic parameters of simplified PESI score, such as age $>80$ years, history of cancer, chronic cardiopulmonary disease, pulse rate $\geq 110$ beats/min, systolic blood pressure $<100$ $\mathrm{mmHg}$ and arterial oxyhemoglobin saturation $<90 \%$. ROC analysis was performed to define a cut-off value for AST/ALT ratio to predict death with appropriate sensitivity and specificity. A twosided p-value $<0.05$ was considered statistically significant. Statistical analyses were performed with the SPSS software (version 15.0 for Windows; SPSS Inc., Chicago, IL, USA).

\section{RESULTS}

The baseline characteristics of 164 study patients are summarised in Table I. The mean age of all patients was $64 \pm 16$ years; and of those, 75 were males (46\%). Patients who died in the hospital were significantly older than patients who survived; and were safely discharged (74 \pm 13 vs. $62 \pm 16, p$ $<0.001$ ). History of cancer was found in 19 of all patients in the study, which was significantly more frequent in deceased patients ( $n=12$ vs. $7, p<0.001)$. There was no other statistically significant difference between groups in terms of demographic data. All patients had contrast-enhanced computed tomography showing the level of thrombus in their pulmonary arterial bed (main, lobar, segmental, or subsegmental). The location of the thrombus was not related to death in the analysis of each segment. Overall, 33 (20\%) patients were recorded as deceased in-hospital in the study cohort. Patients with primary outcome had higher troponin levels in comparison with those who were alive at the time of discharge from the hospital $\left(0.034 / 0.165 / 0.321\right.$ vs. $0.006 / 0.032 / 0.171,25^{\text {th }} / 50^{\text {th }} / 75^{\text {th }}$ percentiles, $p=0.007$ ). Patients who died in hospital were more likely to present with lower systolic blood pressures with a median value of $108 \mathrm{~mm} \mathrm{Hg}$ vs. $112 \mathrm{mmHg}$, respectively $\left(25^{\text {th }}-75^{\text {th }}\right.$ percentiles, $90-111 \mathrm{mmHg}$ vs. $107-130 \mathrm{~mm} \mathrm{Hg}$, respectively, $p<0.001$, Table I). RV diameter to left ventricle (LV) diameter ratio was higher in patients who were dead, but this did not reach statistical significance $(0.96 \pm 0.22$ vs. 0.91 $\pm 0.19, \mathrm{p}=0.2$ ).

AST/ALT ratio was higher in patients who were dead in hospital (median, $1.4 ; 25^{\text {th }}-75^{\text {th }}$ percentile, $\left.1.1-1.8\right)$ comparing to patients who were discharged home (median, $1.1 ; 25^{\text {th }}-75^{\text {th }}$ percentile, 0.84-1.4) ( $p=0.006$, Table I). There was a positive correlation between AST/ALT ratio and SPESI score $(r=0.287$, $p=0.005$ ). The receiver operating characteristics curve further revealed that AST/ALT ratio was an indicator of in-hospital mortality in patients with acute PE. The calculated area under the curve was 0.655 for AST/ALT ratio $(95 \% \mathrm{Cl}, 0.547-0.764)$ (Figure 1). 
Table I: Baseline demographics, laboratory markers and vital measurements of the study group; and their relationship with in-hospital mortality.

\begin{tabular}{|c|c|c|c|c|}
\hline & $\begin{array}{l}\text { All study Group } \\
(\mathrm{N}=164)\end{array}$ & $\begin{array}{c}\text { Deceased } \\
(\mathrm{N}=33)\end{array}$ & $\begin{array}{c}\text { Discharged } \\
(\mathrm{N}=131)\end{array}$ & $\mathbf{P}$ \\
\hline $\begin{array}{l}\text { Age (years) } \\
\text { Mean (SD) }\end{array}$ & $64(16)$ & $74(13)$ & $62(16)$ & $<0.001^{\mathrm{b}}$ \\
\hline $\operatorname{Sex}(M / F)$ & $74 / 90$ & $14 / 19$ & $60 / 71$ & $0.7^{b}$ \\
\hline $\mathrm{BMI}^{\mathrm{a}}, \mathrm{kg} / \mathrm{m} 2$ & $28(25-31)$ & $28(24-31)$ & $28(25-30)$ & $0.8^{c}$ \\
\hline $\mathrm{DM}, \mathrm{n}(\%)$ & $44(27)$ & $11(33)$ & $33(20)$ & $0.5^{b}$ \\
\hline HT, n (\%) & $71(43)$ & $12(36)$ & $59(36)$ & $0.2^{\mathrm{b}}$ \\
\hline Renal Failure, n (\%) & $10(6)$ & $2(6)$ & $8(6)$ & $1^{\mathrm{b}}$ \\
\hline CAD, n (\%) & $18(10)$ & $2(6)$ & $16(12)$ & $0.4^{\mathrm{b}}$ \\
\hline Heart Failure, n (\%) & 19(11) & $4(12)$ & $15(11)$ & $1^{\mathrm{b}}$ \\
\hline History of Cancer, n (\%) & 19(11) & $12(36)$ & $7(5)$ & $<0.001^{b}$ \\
\hline COPD, n (\%) & $21(12)$ & $3(9)$ & $18(10)$ & $0.6^{\mathrm{b}}$ \\
\hline \multirow[t]{2}{*}{$\begin{array}{l}\text { History of immobilisation, } \mathrm{n} \\
(\%)\end{array}$} & $51(31)$ & $16(48)$ & $35(26)$ & $0.06^{b}$ \\
\hline & $\begin{array}{c}\text { All patients } \\
(n=164)\end{array}$ & $\begin{array}{c}\text { Deceased } \\
(n=33)\end{array}$ & $\begin{array}{c}\text { Discharged } \\
(n=131)\end{array}$ & $\mathbf{p}$ \\
\hline Troponin $^{\text {a }}$ (ng/mL) & $0.052(0.006-0.211)$ & $0.165(0.034-0.3219$ & $0.032(0.006-0.171)$ & $0.007^{b}$ \\
\hline $\mathrm{SaO} 2<90 \%, \mathrm{n}(\%)$ & $37(22 \%)$ & $11(33 \%)$ & $26(19 \%)$ & $0.04^{c}$ \\
\hline Heart rate (bpm) mean (SD) & $93(21)$ & $100(27)$ & $91(18)$ & $0.02^{d}$ \\
\hline $\mathrm{SBP}^{\mathrm{a}}(\mathrm{mmHg})$ & $110(100-120)$ & $108(90-111)$ & $112(107-130)$ & $0.001^{b}$ \\
\hline $\mathrm{DBP}^{\mathrm{a}}(\mathrm{mmHg})$ & $70(60-80)$ & $60(60-70)$ & $70(63-80)$ & $<0.001^{\mathrm{b}}$ \\
\hline AST/ALT ratio ${ }^{a}$ & $1.2(0.9-1.5)$ & 1.4(1.1-1.8) & $1.1(0.8-1.4)$ & $0.006^{b}$ \\
\hline D-dimer ${ }^{\mathrm{a}}(\mathrm{mg} / \mathrm{L})$ & $4502(2350-8000)$ & $4113(2605-9900)$ & $4601(2350-8000)$ & $0.9^{b}$ \\
\hline PESI score ${ }^{a}$ & $1(0-2)$ & $2(1-2.25)$ & $1(0-2)$ & $<0.001^{b}$ \\
\hline
\end{tabular}

Table II: Binary logistics regression analysis showing independent predictors of in-hospital mortality.

\begin{tabular}{|l|l|l|l|}
\hline & OR & $\mathbf{9 5 \%} \mathbf{~ C l}$ & $\mathbf{p}$ \\
\hline Troponin & 1.46 & $0.84-2.53$ & 0.17 \\
\hline History of cancer & 30.28 & $5.56-164.84$ & $<0.001$ \\
\hline Age $>80$ & 81.11 & $1.52-43.08$ & 0.014 \\
\hline SBP <100mm Hg & 2.60 & $0.55-12.16$ & 0.22 \\
\hline HR $>100$ & 4.37 & $1.15-16.56$ & 0.03 \\
\hline AST/ALT ratio & 1.12 & $0.40-3.11$ & 0.82 \\
\hline $\begin{array}{l}\text { ALT: Alanine aminotransferase; AST: Aspartate aminotransferase; } \\
\text { rate; SBP: } \text { Heart }\end{array}$ \\
\hline
\end{tabular}

The cut-off value of 1.3 was associated with a prognostic sensitivity of $61 \%$ and specificity of $65 \%$. Binary logistic regression analysis, including troponin and AST/ALT values, revealed that neither of these variables was independently associated with hospital all-cause mortality in our study group (Table II). Age $>80$, heart rate $>110 /$ min, and history of cancer were independent predictors of in-hospital all-cause mortality (OR, 8.11[1.52 - 43.08], 4.37[1.15 - 16.56] and $30.28[5.56-164.84]$, respectively).

\section{DISCUSSION}

The main findings from the study can be briefly outlined as AST/ALT ratio being an indicator of in-hospital mortality in PE patients with a cut-off value of 1.3. AST/ALT ratio and troponin levels were not found to be an independent predictor of all-cause in-hospital mortality according to binary logistic regression analysis.

AST/ALT ratio and transaminase levels have recently been studied as a prognostic marker in acute myocardial infarction, and it was found to be strongly associated with longterm mortality and an independent predictor of cardiovascular mortality. ${ }^{12}$ Another study from Gao et al. investigated the prognostic significance of the AST/ALT ratio in a retrospective cohort of 421 acute ischemic stroke patients. It revealed that a cut-off level of 1.53 for AST/ALT ratio was associated with poor outcome at three months with $29.5 \%$ sensitivity and $85.3 \%$ specificity. ${ }^{13}$ These findings from previous trials are consistent with our findings that the AST/ALT ratio is an indicator of poor prognosis in acute PE.

Another interesting study evaluated the predictive property of AST/ALT values to determine the risk of atrial fibrillation (AF) in a particular population of celiac disease patients. ${ }^{14}$ In this study, patients were divided into two groups as fibrosis-prone and non-fibrosis prone, according to AST/ALT ratio, which is used as a marker for liver fibrosis in celiac disease. Fibrosis-prone patients seem to be at high risk of developing AF using some surrogate electrophysiological markers of 
atrial fibrosis and AF. As a marker of fibrosis in the liver, AST/ALT ratio is not directly linked to atrial fibrosis and AF, so in this study, it is not clear whether it is just showing the risk of systemic inflammation and fibrosis in this specific patient group. A meta-analysis of prospective studies assessing liver enzymes AST, ALT, alkaline phosphatase (ALP), and gamma-glutamyl transferase (GGT) as predictors of CVD used a combined endpoint of CVD, stroke, and cardiovascular death. ${ }^{15}$ This meta-analysis has reported that ALP and GGT but not AST or ALT are positively and independently associated with cardiovascular risk. AST/ALT ratio was not included in this analysis.

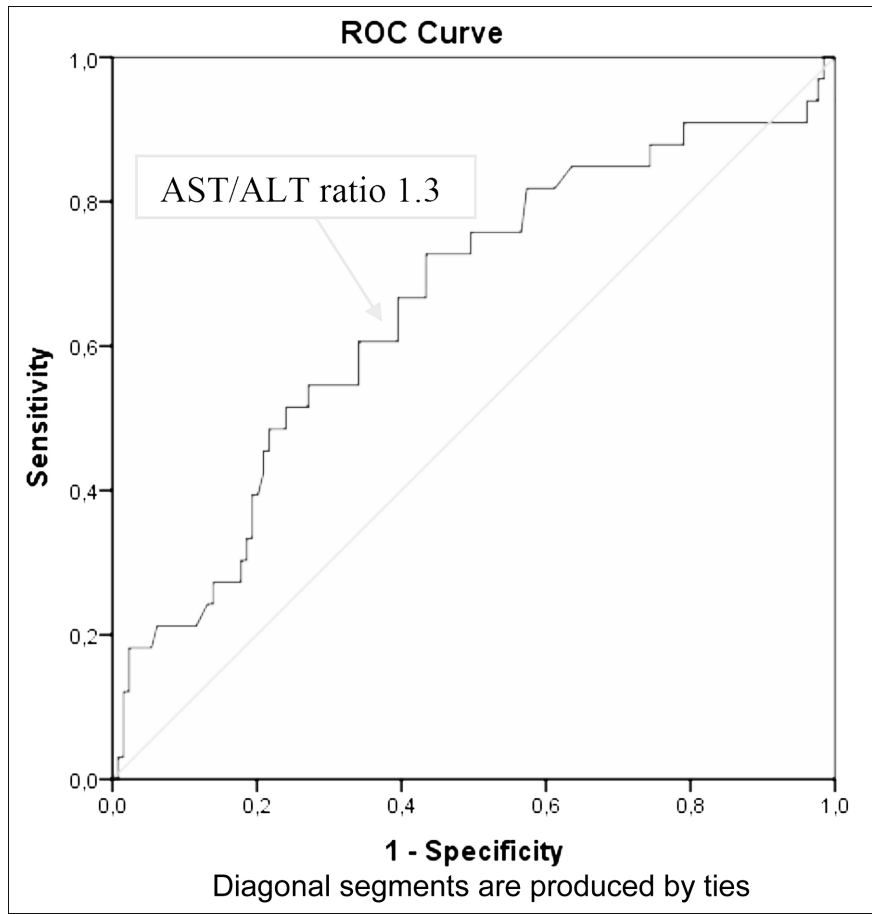

Figure 1: Receiver operating characteristics curve showing the prognostic sensitivity and specificity of AST/ALT ratio with regard to inhospital mortality.

Poelzl et al. reported in their study that in the case of acute heart failure, serum transaminases rise predominantly in the serum, whereas chronic heart failure is mainly related to the elevation of cholestatic enzymes. ${ }^{16}$ Van Deursen et al. studied the relationship between right heart catheterisation findings and liver function tests in 323 heart failure patients. ${ }^{17}$ When they stratified their patients according to their cardiac index $(\mathrm{Cl})$ and central venous pressure (CVP), they revealed that high CVP was related to elevation of most of the liver function tests, including alkaline phosphatase and bilirubin levels but only rise of AST, ALT and total bilirubin levels were associated with reduced $\mathrm{Cl}$. Both of these studies revealed that serum transaminase levels tend to increase specifically in acute heart failure clinics with reduced cardiac output.

A longitudinal cohort study from Yokoyoma et al. prospectively investigated the relationship between AST/ALT ratio and mortality from CVD in 3,494 Japanese subjects, which they have followed for ten years. ${ }^{18}$ In that study, the AST/ALT ratio was an independent predictor of all-cause and CV mortality but not noncardiovascular mortality. When they divided their patient group into high and low AST/ALT ratio categories, there was a significantly higher number of patients with a history of CVD in the high AST/ALT ratio group than the low AST/ALT ratio group. There was no significant difference in the history of CVD in patients cohort between patients who were deceased in hospital and discharged patients. A retrospective cohort analysis on peritoneal dialysis patients similarly revealed that baseline high AST/ALT ratio in these patients is associated with increased CV mortality. ${ }^{19}$ These studies document the long-term predictive value of the AST/ALT ratio for CV mortality.

In PE, an acute increase in PAP will directly lead to elevated hepatic venous pressure and a decrease in porto-hepatic venous gradient and blood flow. On the other hand, accompanying fall in $\mathrm{CO}$ will decrease hepatic artery blood flow and decrease the global blood supply of the liver. These acute changes in local hemodynamics will lead to zone 3 necrosis in the liver, where AST exists in higher concentrations than zones 1 and $2 .{ }^{20}$ Even in hemodynamically stable PE patients, reduced CO may lead to zone 3 ischemia in the liver and a rise in serum transaminase levels with the mechanism as mentioned earlier. This association between reduction of $\mathrm{CO}$ and concomitant hypoperfusion of liver in PE might explain our study findings and linear relationship between rising AST/ALT ratio levels and early poor outcome. Accordingly, Kostrubiec et al. studied glomerular filtration rate (GFR) in acute PE patients as the decrease in CO directly leads to a decrease in renal perfusion and affects GFR levels. ${ }^{21}$ They concluded that in initially normotensive PE patients, a GFR $<35 \mathrm{~mL} / \mathrm{min}$ predicts 30 -day mortality.

Moreover, AST is also a myocardial enzyme, and in myocardial cell death, the AST level is expected to rise in serum. It used to be part of a routine blood chemistry investigation in myocardial infarction patients before troponin and CK-MB era. ${ }^{22}$ Therefore, both myocardial and hepatic ischemia during the course of PE might explain the relationship of increased AST/ALT ratio with in-hospital mortality in our study. As far as the authors know, this is the first study in the literature assessing the prognostic significance of the AST/ALT ratio in PE patients.

This study is a retrospective analysis in a small patient group. The relatively low number of patients included in our study might have affected the ROC analysis and regression analysis results for the AST/ALT ratio. Troponin, a well-described prognostic marker of early death in PE patients, was not an independent predictor of in-hospital death in the study either. On the other hand, there was a positive correlation between the SPESI scores and the AST/ALT ratio values of patients. These findings might point out the need for prospective studies with more patients to reach a definite conclusion on the prognostic significance of AST/ALT ratio as an independent predictor. 


\section{CONCLUSION}

AST/ALT ratio with a cut-off value of 1.3 , measured at the time of diagnosis in PE patients, might predict in-hospital death with acceptable sensitivity and specificity. This finding might contribute to physicians for risk stratification in acute PE patients in the emergency settings.

\section{ETHICAL APPROVAL:}

This study was approved by the Sakarya University School of Medicine Institutional Ethics Committee. (Approval date and No. 27.07.2020/430).

\section{PATIENTS' CONSENT:}

All patients signed written informed consent.

\section{CONFLICT OF INTEREST:}

The authors declared no conflict of interest.

\section{AUTHORS' CONTRIBUTION:}

MNMA: Conception and design of the study, interpretation of the analysed data and writing of the manuscript, and final approval of the study.

FT, IS: Patient selection, data acquisition, data analysis, and final approval of the study.

SA: Design and conception of the study, interpretation of the data, and final approval of the study.

\section{REFERENCES}

1. Raskob GE, Angchaisuksiri P, Blanco AN, Buller H, Gallus A, Hunt BJ, et al. Thrombosis: A major contributor to global disease burden. Arterioscler Thromb Vasc Biol 2014; 34(11):2363-71. doi: 10.1161/ATVBAHA.114.304488.

2. Harjola VP, Mebazaa A, Celutkiene J, Bettex D, Bueno H, Chioncel $\mathrm{O}$, et al. Contemporary management of acute right ventricular failure: A statement from the heart failure association and the working group on pulmonary circulation and right ventricular function of the european society of cardiology. Eur J Heart Fail 2016; 18(3):226-41. doi: 10.1002/ejhf.478.

3. Lankeit M, Jimenez D, Kostrubiec M, Dellas C, Hasenfuss G, Pruszczyk $P$, et al. Predictive value of the high-sensitivity troponin $T$ assay and the simplified Pulmonary Embolism Severity Index in hemodynamically stable patients. With acute pulmonary embolism: A prospective validation study. Circulation 2011; 124(4):2716-24. doi: 10.1161/CIRCULATIONAHA.111.051177.

4. Lankeit M, Kempf T, Dellas C, Cuny M, Tapken H, Peter T, et al. Growth differentiation factor-15 for prognostic assessment of patients with acute pulmonary embolism. Am J Respir Crit Care Med 2008; 177(9):1018-25. doi: 10.1164/rccm.200712-17860C.

5. Klok FA, Mos IC, Huisman MV. Brain-type natriuretic peptide levels in the prediction of adverse outcome in patients with pulmonary embolism: A systematic review and meta-analysis. Am J Respir Crit Care Med 2008; 178(4):425-43. doi: 10.1164/rccm.200803-4590C.

6. Coutance G, Cauderlier E, Ehtisham J, Hamon M, Hamon M.
The prognostic value of markers of right ventricular dysfunction in pulmonary embolism: A meta-analysis. Crit Care 2011; 15(2): R103. doi: 10.1186/cc10119.

7. Sanchez O, Trinquart L, Colombet I, Durieux P, Huisman MV, Chatellier G, et al. Prognostic value of right ventricular dysfunction in patients with haemodynamically stable pulmonary embolism: A systematic review. Eur Heart J 2008; 29(12):1569-77. doi: 10.1093/eurheartj/ehn208.

8. Kaeberich A, Seeber V, Jimenez D, Kostrubiec M, Dellas C, Hasenfuss G, et al. Age adjusted high- sensitivity troponin $T$ cut-off value for risk stratification of pulmonary embolism. Eur Respir J 2015; 45(5):1323-31. doi: 10.1183/ 09031936.00174514.

9. Jiménez D, Aujesky D, Moores L, Gómez V, Lobo JL, Uresandi $F$, et al. RIETE Investigators. Simplification of the pulmonary embolism severity index for prognostication in patients with acute symptomatic pulmonary embolism. Arch Intern Med 2010; 170(15):1383-9. doi: 10.1001/archinternmed.2010.199.

10. Aujesky D, Obrosky DS, Stone RA, Auble TE, Perrier A, Cornuz J, et al. Derivation and validation of a prognostic model for pulmonary embolism. Am J Respir Crit Care Med 2005; 172(8):1041-6. doi: 10.1164/rccm.200506-8620C.

11. Correale M, Tarantino N, Petrucci R, Tricarico L, Laonigro I, Di Biase $M$, et al. Liver disease and heart failure: Back and forth. Eur J Intern Med 2018; 48:25-34. doi: 10.1016/j.ejim.2017.10.016.

12. Steininger $M$, Winter MP, Reiberger T, Koller L, El-Hamid F, Forster $\mathrm{S}$, et al. De-ritis ratio improves long-term risk prediction after acute myocardial infarction. J Clin Med 2018; 7(12):474. doi: 10.3390/jcm7120474.

13. Gao F, Chen C, Lu J, Zheng J, Ma XC, Yuan XY, et al. De Ritis ratio (AST/ALT) as an independent predictor of poor outcome in patients with acute ischemic stroke. Neuropsychiatr Dis Treat 2017; 13:1551-7. doi: 10.2147/NDT.S139316.

14. Kucukseymen S, Cekin AH, Bayar N, Arslan S, Uygur Kucukseymen $\mathrm{E}$, Mercan $\mathrm{T}$, et al. A novel biomarker for prediction of atrial fibrillation susceptibility in patients with celiac disease. PLoS One 2018; 13(1):e0190382. doi: 10.1371/ journal.pone.0190382.

15. Kunutsor SK, Apekey TA, Khan H. Liver enzymes and risk of cardiovascular disease in the general population: A meta-analysis of prospective cohort studies. Atherosclerosis 2014; 236(1):7-17. doi: 10.1016/j.atherosclerosis.2014.06.006.

16. Poelzl G, Ess M, Mussner-Seeber C, Pachinger O, Frick M, Ulmer $\mathrm{H}$. Liver dysfunction in chronic heart failure: Prevalence, characteristics and prognostic significance. Eur J Clin Invest 2012; 42(2):153-63. doi: 10.1111/j.1365-2362. 2011.02573.x.

17. van Deursen VM, Damman K, Hillege HL, van Beek AP, van Veldhuisen DJ, Voors AA. Abnormal liver function in relation to hemodynamic profile in heart failure patients. J Card Fail 2010; 16(1):84-90. doi: 10.1016/j.cardfail.2009.08.002.

18. Yokoyama M, Watanabe T, Otaki Y, Takahashi H, Arimoto T, Shishido $\mathrm{T}$, et al. Association of the aspartate aminotransferase to alanine aminotransferase ratio with BNP Level and cardiovascular mortality in the general population: The 
yamagata study 10-year follow-up. Dis Markers 2016; 2016:4857917. doi: 10.1155/2016/4857917.

19. Feng $X$, Wen $Y$, Peng FF, Wang $N$, Zhan X, Wu X. Association between aminotransferase/alanine aminotransferase ratio and cardiovascular disease mortality in patients on peritoneal dialysis: A multi-centre retrospective study. $B M C$ Nephrol 2020; 21(1):209. doi: 10.1186/s12882-02001840-7.

20. Giannini EG, Testa R, Savarino V. Liver enzyme alteration: A guide for clinicians. CMAJ 2005; 172(3):367-79. doi: 10.1503/cmaj.1040752.

21. Kostrubiec M, Łabyk A, Pedowska-Włoszek J, Pacho S, Wojciechowski $A$, Jankowski $K$ et al. Assessment of renal dysfunction improves troponin-based short-term prognosis in patients with acute symptomatic pulmonary embolism. J Thromb Haemost 2010; 8(4):651-8. doi: 10.1111/j. 1538-7836.2010.03762.x.

22. Lofthus DM, Stevens SR, Armstrong PW, Granger CB, Mahaffey KW. Pattern of liver enzyme elevations in acute ST-elevation myocardial infarction. Coron Artery Dis 2012; 23(1):22-30. doi: 10.1097/MCA.0b013e32834e4ef1. 